\title{
Exploring soil microbial communities: Opportunities for soil ecology research
}

\author{
James I. Prosser \\ School of Biological Sciences, University of Aberdeen, Cruickshank Building, Aberdeen AB24 3UU, United Kingdom \\ (c) Higher Education Press 2019
}

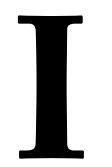

$\mathrm{t}$ is a pleasure to contribute, with this editorial, to the first issue of Soil Ecology Letters. My own interests in soil ecology lie in understanding the mechanisms determining microbial community composition and its importance for soil ecosystem functions. Microorganisms obviously have crucial and central roles in soil formation and sustainability, in the full range of biogeochemical cycling processes, in facilitating crop growth and, also, as plant pathogens. Microbial community ecology is therefore an important topic but, until the early 1990s, we relied on cultivation-based techniques to study microbial communities. Then we witnessed major technical advances, through the development of molecular techniques that enable relatively rapid and relatively cheap characterisation of communities in natural environments, including the soil. While these techniques are not perfect, they avoid the major limitation of the requirement for laboratory cultivation and the unavoidable dangers of selection for 'laboratory rats' or 'domesticated' organisms that are not representative of natural communities. Early studies, in which soil microorganisms were characterised by amplification and sequencing of 16S rRNA genes, indicated that concerns over cultivation bias were justified. They provided strong evidence for much higher richness than observed in laboratory cultures, the presence of 'novel', but abundant, high level taxonomic groups with no cultivated representatives and the existence of novel groups within phyla that were previously considered to be well characterised. These findings rapidly became 'the norm', and we would now be very surprised to find a soil with low microbial diversity or to isolate easily microorganisms that were abundant in the soil.

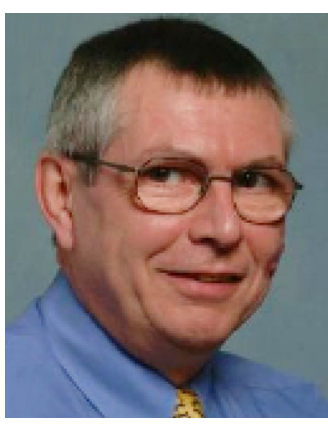

Prof. James I. Prosser

The introduction of new techniques in microbial ecology, and in other fields, frequently leads to many descriptive studies and surveys. This has certainly proved to be the case for soil microbial communities, with innumerable papers describing sequences of 16S rRNA genes and functional genes that tell us 'who is there'. These are now often accompanied by further descriptions of what the organisms might be able to do or, less frequently, what they are doing. These techniques employ a range of ways in which the physiology, activity and function of organisms can be inferred without cultivation in the laboratory and increasingly include metagenomic, metatranscriptomic and other omics-based approaches.

Soil microbial community ecology has therefore addressed technical challenges but much less attention has been paid to scientific challenges. By this I mean that, while we have greatly increased our ability to determine who is there and, sometimes, what they are doing, these descriptive studies have rarely increased our understanding of, for example, why particular organisms are in particular soils, the factors and mechanisms that determine community assembly and composition, whether, when and how community composition (as opposed to microbial biomass) significantly influences ecosystem function and how and why communities and their activities might respond to environmental change. In other words, there are many studies of their natural history but these have rarely contributed to advances in knowledge and understanding of their ecology. As a consequence, despite considerable investment in soil microbial community analyses, we have not significantly increased our ability to predict community composition, dynamics, resilience or resistance or the consequences of community composition for process rates or 
our ability to manipulate microbial communities, e.g. to increase plant productivity. In fact, many studies actually highlight our lack of understanding and ignorance of soil microbial ecology.

The good news is that the major limitation in addressing these scientific challenges is not a lack of suitable techniques, but a need to adopt a different approach. For example, the majority of studies do not address specific or important scientific or ecological questions and the vast majority are not driven by ideas, concepts or hypotheses based on assumptions regarding underlying mechanisms. As a consequence, studies tend to be unstructured, lack clear frameworks or objectives, lack generality and lack a theoretical or conceptual basis. A further problem is the dominance of studies in which community composition is correlated with a small number of soil characteristics, chosen on the basis of low cost and ease of measurement. While such studies sometimes identify correlations or patterns, they rarely increase understanding because of their inability to distinguish cause and effect, the irrelevance of measured soil characteristics to potential mechanisms, lack of knowledge of links between phylogeny and physiology and the unavoidable bias in explanations based on post hoc interpretation of data.

Descriptive and correlation-based, pattern-seeking studies will doubtless continue but real advances will only arise by identifying important scientific and ecological questions and addressing them using scientific approaches, in which mechanism-based ideas and hypotheses are developed to explain phenomena and then tested in carefully designed experiments. The complexity of soil ecosystems makes such approaches even more important and reduces the likelihood of unstructured, correlation-based studies providing meaningful answers.

There are therefore major opportunities for a new soil ecology journal. Soil microorganisms cannot be ignored by soil ecologists and understanding and predicting their diversity and ecosystem function is one of the most exciting areas in ecological research. Hopefully Soil Ecology Letters will play a role in encouraging and highlighting experimental, hypothesis-driven studies that harness the power of molecular techniques for community analysis, and other relevant techniques, to address the important ecological questions in soil microbial community ecology.

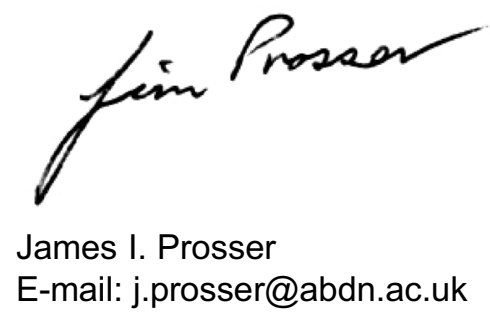

